\title{
Digital subtraction angiography vs. real-time fluoroscopy for detection of intravascular injection during transforaminal epidural block
}

\author{
Kibeom Park ${ }^{1}$, Saeyoung Kim ${ }^{2}$ \\ ${ }^{1}$ Department of Anesthesiology and Pain Medicine, Keimyung University School of Medicine, Daegu, Korea \\ ${ }^{2}$ Department of Anesthesiology and Pain Medicine, Kyungpook National University School of Medicine, Daegu, Korea
}

Received: October 29, 2018

Revised: January 18, 2019

Accepted: January 21, 2019

Corresponding author:

Saeyoung Kim

Department of Anesthesiology and

Pain Medicine, Kyungpook National

University School of Medicine,

130, Dongdeok-ro, Jung-gu,

Daegu 41944, Korea

Tel: $+82-53-200-5873$

Fax: +82-53-426-2760

E-mail: saeyoungkim7@gmail.com
Background: Transforaminal epidural block (TFEB) is an effective treatment option for radicular pain. To reduce complications from intravascular injection during TFEB, use of imaging modalities such as real-time fluoroscopy (RTF) or digital subtraction angiography (DSA) has been recommended. In this study, we investigated whether DSA improved the detection of intravascular injection during TFEB at the whole spine level compared to RTF.

Methods: We prospectively examined 316 patients who underwent TFEB. After confirmation of final needle position using biplanar fluoroscopy, $2 \mathrm{~mL}$ of nonionic contrast medium was injected at a rate of $0.5 \mathrm{~mL} / \mathrm{s}$ under RTF; $30 \mathrm{~s}$ later, $2 \mathrm{~mL}$ of nonionic contrast medium was injected at a rate of $0.5 \mathrm{~mL} / \mathrm{s}$ under DSA.

Results: Thirty-six intravascular injections were detected for an overall rate of 11.4\% using RTF, with 45 detected for a rate of $14.2 \%$ using DSA. The detection rate using DSA was statistically different from that using RTF ( $p=0.004)$. DSA detected a significantly higher proportion of intravascular injections at the cervical level than at the thoracic $(p=0.009)$ and lumbar $(p=0.011)$ levels. Conclusion: During TFEB at the whole spine level, DSA was better than RTF for the detection of intravascular injection. Special attention is advised for cervical TFEB, because of a significantly higher intravascular injection rate at this level than at other levels.

Keywords: Analgesia; Complications; Epidural; Radiculopathy; Spine

\section{Introduction}

Transforaminal epidural block (TFEB) is an effective diagnostic and treatment option for spinal radicular pain [1]. The transforaminal approach is target-specific, compared with other approaches for epidural blocks [2]. Potential risks associated with TFEB include infection $[3,4]$, dural puncture $[5,6]$, bleeding, and intravascular injection [5]. To reduce complications resulting from intravascular injection of drugs, several methods have been proposed, including use of short-beveled or blunt-type needles, large-diameter needles, non-particulate steroids, or imaging modalities such as real-time fluoroscopy (RTF) or digital subtraction angiography (DSA) [7]. There are no case reports or studies about fatal neurologic events resulting from intravascular injection of non-particulate steroids, but administration of local anesthetics may cause rare complications in the central nervous system during a cervical TFEB. Local anesthetics depress respiration and consciousness during a cervical root block [8].

Copyright@ 2019 Yeungnam University College of Medicine

This is an Open Access article distributed under the terms of the Creative Commons Attribution Non-Commercial License (http://creativecommons.org/licenses/by-nc/4.0/) which permits unrestricted non-commercial use, distribution, and reproduction in any medium, provided the original work is properly cited. 
Injection of a local anesthetic through a vertebral artery can cause loss of consciousness and seizures [9].

RTF reportedly failed to detect $29.0 \%$ of intravascular injections compared to DSA during lumbosacral TFEB [10]. However, Kim et al. [11] found no benefit with use of DSA compared to RTF during lumbosacral TFEB. DSA has disadvantages such as increased radiation exposure to the physician and patient and high cost of equipment compared to RTF [12]. DSA for TFEB was reported to increase the effective radiation dose by 2.3- to 4.3-fold compared to conventional fluoroscopy [13].

To our knowledge, no report has prospectively compared DSA and RTF during TFEB at the whole spine level, including cervical, thoracic, lumbar, and sacral levels, in the same patient.

The present study investigated whether DSA improved the detection rate for intravascular injection during TFEB at the whole spine level, compared to that using RTF.

\section{Materials and methods}

\section{Patients and exclusion criteria}

The present study was approved by the Institutional Review Board of our hospital (DSMC 2015-09-042), and informed written consent was obtained from all participants.

We prospectively examined 316 TFEB procedures. Inclusion criteria were age over 18 years and radicular pain from herniated nucleus pulposus, spinal stenosis, post-spinal surgery syndrome; zoster-induced pain; or pain owing to other conditions such as complex regional pain syndrome. Exclusion criteria were pregnancy, allergy to contrast medium and local anesthetics, participant refusal, and persistent contraindication to epidural block such as coagulopathy and infection at the injection site.

\section{Intervention and data collection}

Two pain-management physicians were involved in this study. Both physicians were board-certified in the department of pain medicine, and had more than 8 years of working experience. TFEB was performed by one physician and simultaneously observed by the other physician.

Before the procedure, all participants were monitored with an electrocardiogram, pulse oximetry, and noninvasive blood pressure measurement. A 20- or 22-G cannula was inserted in the hand. The participants did not receive sedation. Under fluoroscopic guidance, TFEB was performed using a Quincke type, 25-G, 9-cm spinal needle (Taechang Industrial Co., Kongju, Korea). For cervical level injection, the participant was placed in a supine position on a table with the head slightly extended. The fluoroscope (Ziehm Vision, Ziehm Imaging, Nuremberg,
Germany) was rotated obliquely $45-55^{\circ}$ to the ipsilateral side to provide the best view of the selected neural foramen. The needle was advanced to the superior articular process, at the division between the caudal third and middle third. The needle was then advanced into the neural foramen, touching its posterior border to the halfway point between the medial and lateral borders of the articular pillars in an anteroposterior $(\mathrm{AP})$ view. For thoracic level injection, the participant was placed in prone position. The fluoroscopic beam was aligned perpendicular to the vertebral endplates in an $\mathrm{AP}$ view and then rotated to a $10-20^{\circ}$ oblique angle towards the side being injected. The needle was advanced from a point between the lateral margin of the pedicle and the medial aspect of the rib head to the posterior surface of the vertebral body using tunnel vision technique. For lumbar injections, the participant was placed in prone position. The fluoroscope was tilted in the caudocephalad direction to align parallel with the endplates in an AP view. The fluoroscope was rotated to a $20-30^{\circ}$ oblique angle toward the side being injected, to bring the "Scotty dog" appearance of the spine into view. The needle was advanced into the neural foramen at a point just below the "chin" of the "Scotty dog" with tunnel vision technique. For sacral injections, the participant was placed in prone position. The fluoroscope was tilted in the caudocephalad direction to align parallel with the L5 inferior endplate and the S1 superior endplate in an AP view. The needle was advanced to the superior lateral quadrant of the neural foramen.

After confirmation of final needle position using biplanar fluoroscopy, $2 \mathrm{~mL}$ of nonionic contrast medium (Omnipaque 300, GE Healthcare, Little Chalfont, Buckinghamshire, UK) was injected at a rate of $0.5 \mathrm{~mL} / \mathrm{s}$ under RTF; $30 \mathrm{~s}$ later, another $2 \mathrm{~mL}$ of nonionic contrast medium was injected at a rate of 0.5 $\mathrm{mL} / \mathrm{s}$ under DSA. Intravascular injection was defined as contrast medium spreading out through the vascular channel during injection under RTF and DSA. If intravascular injection was observed, the needle position was changed. A total of $2 \mathrm{~mL}$ of $0.5 \%$ lidocaine mixed with dexamethasone $5 \mathrm{mg}$ was injected after intravascular injection was ruled out.

\section{Sample size}

In a previous study, the incidence of intravascular injection during TFEB at the whole spine level was $10.5 \%$ [14]. We considered a $50 \%$ increase in the incidence of intravascular injection to be clinically important. The sample size was estimated with the requirement of $<0.05$ and $<0.2$ Type I and II error rates, respectively. Considering a $10 \%$ dropout rate, 316 TFEB cases in each group was required. A flow diagram of this study design is shown in Fig. 1. 


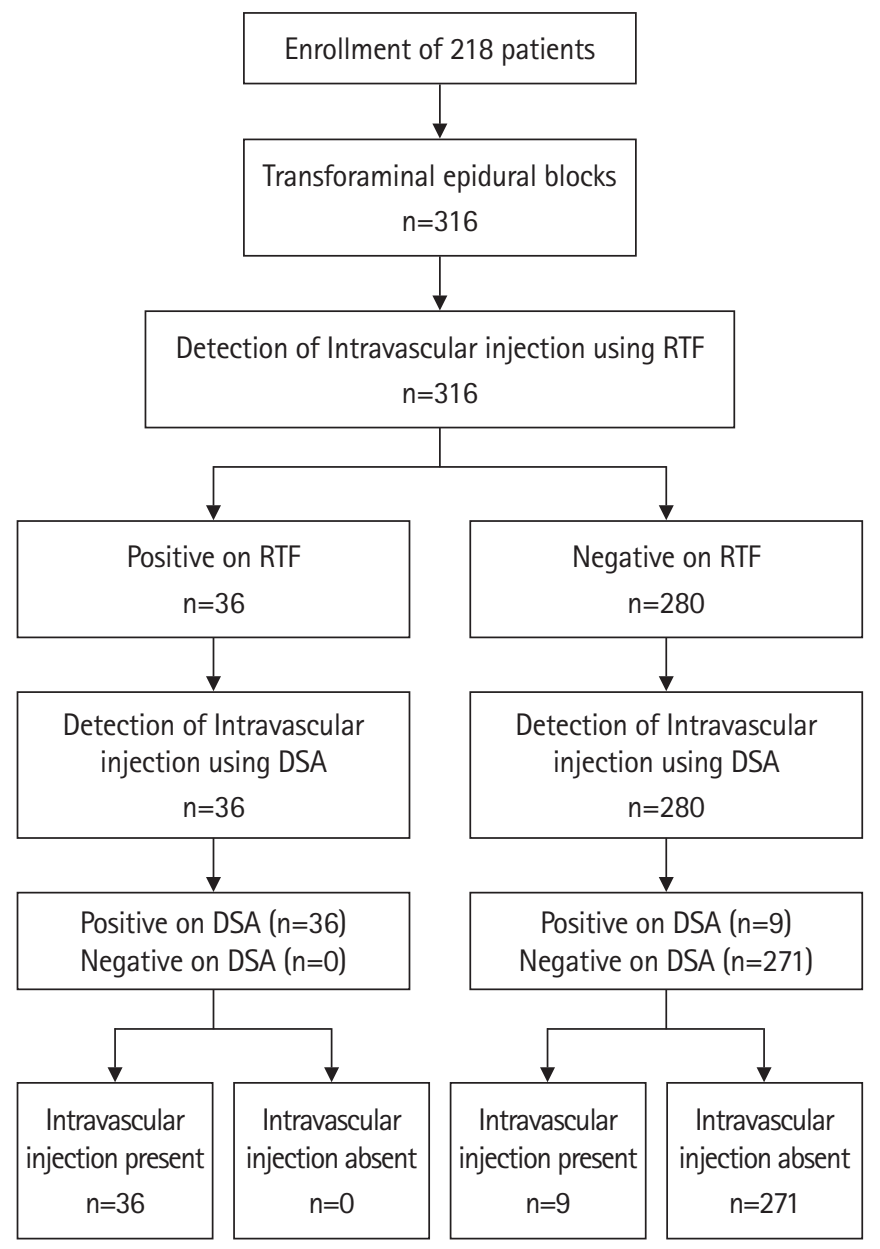

Fig. 1. Flow diagram of the study design. DSA, digital subtraction angiography; RTF, real-time fluoroscopy.

\section{Statistical analysis}

Data on the age, sex, diagnosis, spinal level, and procedure side were collected. The data were analyzed with McNemar's test, using SAS software version 9.3 (Cary, NC, USA). The influence of factors associated with intravascular injection during TFEB was examined using logistic regression analysis, and the adjusted odds ratio (OR) and $95 \%$ confidence interval (CI) were also calculated. A $p$-value $<0.05$ was considered statistically significant.

\section{Results}

In total, 316 TFEB treatments were performed, with 56 injections $(17.7 \%)$ at cervical levels, $31(10.0 \%)$ at thoracic levels, 135 (42.7\%) at lumbar levels, and $94(29.7 \%)$ at sacral levels. There were no complications associated with TFEB. The 316 TFEB treatments were performed in 218 enrolled participants, with a mean age of 62.1 years. The characteristics of study participants are presented in Table 1. TFEB treatments were performed from
Table 1. Characteristics of 316 injections performed in 218 participants

\begin{tabular}{lc}
\hline Variable & Value \\
\hline Age (yr) & $62.14 \pm 12.23$ \\
Height $(\mathrm{cm})$ & $161.81 \pm 8.32$ \\
Weight $(\mathrm{kg})$ & $61.70 \pm 9.83$ \\
Sex (male/female) & $155 / 161$ \\
Site of injection (right/left) & $157 / 159$ \\
Level & \\
Cervical & 56 \\
Thoracic & 31 \\
Lumbar & 135 \\
Sacral & 94 \\
Diagnosis & \\
Herniated nucleus pulposus & 87 \\
Spinal stenosis & 166 \\
Post-spinal surgery syndrome & 21 \\
Zoster-induced pain & 31 \\
Others & 12 \\
Total & 316 \\
\hline
\end{tabular}

Values are presented ad mean \pm standard deviation or number (proportion).

\section{C3 to S2 spinal levels.}

The incidence of intravascular injection at each level detected with DSA and RTF is presented in Table 2. Thirty-six intravascular injections (12 [21.4\%] at cervical, 2 [6.5\%] at thoracic, 11 [8.1\%] at lumbar, and 11 [11.7\%] at sacral levels) were detected, for an overall intravascular injection rate of $11.4 \%$ using RTF. Fortyfive intravascular injections (14 [25\%] at cervical, 3 [9.7\%] at thoracic, $14[10.4 \%]$ at lumbar, and 14 [14.9\%] at sacral levels) were detected, for an overall intravascular injection rate of $14.2 \%$ using DSA. The intravascular injection detection rate using DSA was statistically different from that using RTF $(p=0.004)$. All intravascular injections detected using RTF were also observed using DSA. RTF missed 9 cases of intravascular injection (2 at cervical, 1 at thoracic, 3 at lumbar, and 3 at sacral levels) that were detected using DSA (RTF sensitivity, 80.0\%).

Table 3 shows the adjusted OR and 95\% CI for each variable during intravascular injection using DSA. Only the spinal level showed a significant association with intravascular injection. The incidence of intravascular injection was significantly higher at the cervical level than at the thoracic $(p=0.009)$ and lumbar $(p=0.011)$ levels. Patient age, sex, height, weight, procedure side, and diagnosis had no effect on the incidence.

\section{Discussion}

Our results indicate that intravascular injection was sequentially detected using RTF and DSA during TFEB at the whole spine 
Table 2. Incidence of intravascular injections during transforaminal epidural block

\begin{tabular}{lcc}
\hline & Number of intravascular injections on DSA (\%) & Number of intravascular injections on RTF (\%) \\
\hline Sex (n) & $21(13.5)$ & $17(11.0)$ \\
Male (155) & $24(14.9)$ & $19(11.8)$ \\
Female (161) & $14(25.0)$ & $12(21.4)$ \\
Level of injection (n) & $3(9.7)$ & $2(6.5)$ \\
$\quad$ Cervical (56) & $14(10.4)$ & $11(8.1)$ \\
Thoracic (31) & $14(14.9)$ & $11(11.7)$ \\
Lumbar (135) & & $18(11.5)$ \\
Sacral (94) & $22(14.0)$ & $18(11.3)$ \\
Side of injection (n) & $23(14.5)$ & \\
Right (157) & & $9(10.3)$ \\
Left (159) & $10(11.5)$ & $19(11.4)$ \\
Diagnosis (n) & $25(15.1)$ & $5(23.8)$ \\
Herniated nucleus pulposus (87) & $5(23.8)$ & $2(6.5)$ \\
Spinal stenosis (166) & $3(9.7)$ & $1(8.3)$ \\
Post-spinal surgery syndrome (21) & $2(16.7)$ & $36(11.4)$ \\
Zoster-induced pain (31) & $45(14.2)^{\mathrm{a})}$ & \\
Others (12) & & \\
Total (316) & & \\
\hline
\end{tabular}

Values are presented as number (\%).

DSA, digital subtraction angiography; RTF, real-time fluoroscopy.

${ }^{a)} p<0.05$ compared with RTF.

Table 3. OR and $95 \% \mathrm{Cl}$ of variables on intravascular penetration using DSA

\begin{tabular}{|c|c|c|}
\hline Variable & $\mathrm{OR}$ & $95 \% \mathrm{Cl}$ \\
\hline \multicolumn{3}{|l|}{ Sex } \\
\hline Male & 1.00 & - \\
\hline Female & 1.26 & $0.90-1.82$ \\
\hline Age & 0.99 & $0.96-1.01$ \\
\hline Height & 1.00 & $0.97-1.04$ \\
\hline Weight & 1.02 & $0.99-1.05$ \\
\hline \multicolumn{3}{|l|}{ Side of injection } \\
\hline Right & 1.00 & - \\
\hline Left & 0.94 & $0.50-1.76$ \\
\hline \multicolumn{3}{|l|}{ Level } \\
\hline Cervical & $2.88^{\mathrm{a})}$ & $1.27-6.54$ \\
\hline Thoracic & 0.93 & $0.25-3.44$ \\
\hline Lumbar & 1.00 & - \\
\hline Sacral & 1.51 & $0.69-3.34$ \\
\hline \multicolumn{3}{|l|}{ Diagnosis } \\
\hline Herniated nucleus pulposus & 1.21 & $0.31-4.73$ \\
\hline Spinal stenosis & 1.66 & $0.47-5.86$ \\
\hline Post-spinal surgery syndrome & 3.11 & $0.65-14.85$ \\
\hline Zoster-induced pain & 1.87 & $0.27-12.85$ \\
\hline Others & 1.00 & \\
\hline
\end{tabular}

$\mathrm{OR}$, odds ratio; $\mathrm{Cl}$, confidence interval; $\mathrm{DSA}$, digital subtraction angiography. ${ }^{a)} p<0.05$ compared with thoracic and lumbar level. level. The overall incidence of intravascular injection was $14.2 \%$ using DSA and $11.4 \%$ using RTF. Nine intravascular injections were missed (2 at cervical, 1 at thoracic, 3 at lumbar, and 3 at sacral levels) with RTF compared to DSA. A 25\% improvement was observed using DSA compared to RTF. Therefore, DSA had a better detection rate for intravascular injection during TFEB.

DSA is a radiological technique that can be used to clearly visualize and distinguish blood vessels from surrounding tissues; this is done by subtracting the pre-contrast image from the postcontrast injection image $[12,15]$. Visnjevac et al. [16] reported the efficacy of DSA in detection of intravascular penetration compared with RTF during TFEB in a recent meta-analysis. They included 1,290 TFEB cases (3.2\% at cervical, $76.3 \%$ at lumbar, and $20.5 \%$ at sacral levels) and demonstrated that DSA showed a $32 \%$ improvement in detection of intravascular penetration during TFEB, compared to that using RTF. However, only 3.2\% of cases in their study were performed at cervical levels and did not include any thoracic cases. Therefore, their study could not represent TFEB at the whole spine level. In contrast, the present study attempted to include more cervical and thoracic TFEB cases (17.7\% at cervical and $10.0 \%$ at thoracic levels).

Even though previous studies demonstrated DSA to be superior to RTF for vascular detection during TFEB, McLean et al. [15] indicated that the main vascular uptake observed using DSA was 
attributed to venous uptake and that DSA did not increase the detection rate of arterial uptake. Moreover, in the study of Lee et al. [10], DSA did not successfully distinguish between arterial and venous injections.

Using RTF, Nahm et al. [14] conducted a large prospective study to evaluate the risk factors associated with intravascular injection in TFEB. They evaluated 2,145 injections performed on 1,088 patients and found a significant difference between cervical $(20.6 \%)$ and sacral (16.5\%) level injections and between lumbar $(6.1 \%)$ and thoracic (8.2\%) level injections. They also found no association between intravascular injection during TFEB and patient characteristics such as age, sex, body mass index, and diagnosis. No prior report has investigated the risk factors associated with intravascular injection using DSA at the whole spine level under various conditions. In the present study, the spinal procedure level was the only significant association identified, consistent with the results of a previous study using RTF [14]. The cervical level (25\%) showed a significantly higher intravascular injection rate than the thoracic $(9.7 \%)$ and lumbar (10.4\%) levels.

The present study also found no difference in intravascular injection rates during TFEB according to the diagnosis, consistent with the results of a previous study using RTF $[11,14]$. We also found no differences in intravascular injection rates between DSA and RTF according to diagnosis.

In this study, we used a Quincke needle which is sharp. Because blunt needles will displace and not penetrate vessels owing to their dull tip $[17,18]$, the use of blunt needles during TFEB has been suggested to avoid intravascular injection of steroids $[7,19]$. Animal studies have shown a reduced incidence of arterial puncture and bleeding with the use of blunt needles compared to that using sharp needles $[17,18]$. Several studies have found that use of blunt needles during lumbar TFEB could reduce intravascular injections and paresthesia compared to those using sharp needles [20-22]. However, Smuck et al. [23] failed to find any benefit with use of blunt needles during lumbosacral TFEB compared to that using sharp needles. This issue remains controversial, and further study is needed to determine whether the use of blunt needles could reduce intravascular injections during TFEB.

Intravascular injection was sequentially detected using RTF and DSA guidance in the present study. This study had some limitations. First, the procedural physician was not blinded to the type of imaging modality used to detect intravascular injection. To minimize confirmation bias and provide homogeneous procedural conditions for TFEB, the same procedural physician performed all 316 injections. Second, RTF and DSA were successively used in the same patient during TFEB. Intravascular injections detected by RTF were also detected by DSA. However, the injected contrast medium and extravasation of blood from intravascular penetration during RTF observation may have affected the detection of intravascular injection during DSA observation. Third, it may be impossible to define a vascular contrast spreading pattern as venous or arterial during epidural TFEB because these patterns were ambiguous despite the use of DSA $[24,25]$. We also could not differentiate between the two vascular patterns. Fourth, the proportions of cervical, thoracic, and lumbar levels in TFEB differed, as these reflected the distribution of patients with spinal pain in the pain clinic. Fifth, structural differences according to vertebral levels or individual spinal disease could affect the results, but further studies are needed to clarify this.

During TFEB at the whole spine level, DSA showed a better detection rate than RTF for intravascular injection. Special attention is recommended for cervical TFEB, which showed an intravascular injection rate that was significantly higher than that at other levels.

\section{Conflicts of interest}

No potential conflicts of interest relevant to this article was reported.

\section{ORCID}

Kibeom Park, https://orcid.org/0000-0002-1432-8301

Saeyoung Kim, https://orcid.org/0000-0003-1650-3385

\section{References}

1. Manchikanti L, Singh V, Pampati V, Falco FJ, Hirsch JA. Comparison of the efficacy of caudal, interlaminar, and transforaminal epidural injections in managing lumbar disc herniation: is one method superior to the other? Korean J Pain 2015;28:11-21.

2. Schaufele MK, Hatch L, Jones W. Interlaminar versus transforaminal epidural injections for the treatment of symptomatic lumbar intervertebral disc herniations. Pain Physician 2006;9:361-6.

3. Goodman BS, Posecion LW, Mallempati S, Bayazitoglu M. Complications and pitfalls of lumbar interlaminar and transforaminal epidural injections. Curr Rev Musculoskelet Med 2008;1:212-22.

4. Waldman SD. Complications of cervical epidural nerve blocks 
with steroids: a prospective study of 790 consecutive blocks. Reg Anesth 1989;14:149-51.

5. Manchikanti L, Malla Y, Wargo BW, Cash KA, Pampati V, Fellows B. Complications of fluoroscopically directed facet joint nerve blocks: a prospective evaluation of 7,500 episodes with 43,000 nerve blocks. Pain Physician 2012;15:E143-50.

6. Berger CW, Crosby ET, Grodecki W. North American survey of the management of dural puncture occurring during labour epidural analgesia. Can J Anaesth 1998;45:110-4.

7. Tiso RL, Cutler T, Catania JA, Whalen K. Adverse central nervous system sequelae after selective transforaminal block: the role of corticosteroids. Spine J 2004;4:468-74.

8. Tofuku K, Koga H, Komiya S. Subdural spread of injected local anesthetic in a selective transforaminal cervical nerve root block: a case report. J Med Case Rep 2012;6:142.

9. Chung SG. Convulsion caused by a lidocaine test in cervical transforaminal epidural steroid injection. PM R 2011;3:674-7.

10. Lee MH, Yang KS, Kim YH, Jung HD, Lim SJ, Moon DE. Accuracy of live fluoroscopy to detect intravascular injection during lumbar transforaminal epidural injections. Korean J Pain 2010;23:18-23.

11. Kim YH, Park HJ, Moon DE. Rates of lumbosacral transforaminal injections interpreted as intravascular: fluoroscopy alone or with digital subtraction. Anaesthesia 2013;68:1120-3.

12. Jasper JF. Role of digital subtraction fluoroscopic imaging in detecting intravascular injections. Pain Physician 2003;6:36972.

13. Maus T, Schueler BA, Leng S, Magnuson D, Magnuson DJ, Diehn FE. Radiation dose incurred in the exclusion of vascular filling in transforaminal epidural steroid injections: fluoroscopy, digital subtraction angiography, and CT/ fluoroscopy. Pain Med 2014; 15:1328-33.

14. Nahm FS, Lee CJ, Lee SH, Kim TH, Sim WS, Cho HS, et al. Risk of intravascular injection in transforaminal epidural injections. Anaesthesia 2010;65:917-21.

15. McLean JP, Sigler JD, Plastaras CT, Garvan CW, Rittenberg JD. The rate of detection of intravascular injection in cervical transforaminal epidural steroid injections with and without digital subtraction angiography. PM R 2009;1:636-42.
16. Visnjevac O, Kim P, Farid-Davari S, Johnson P, Nader ND. Digital subtraction angiography versus real-time fluoroscopy for detection of intravascular penetration prior to epidural steroid injections: meta-analysis of prospective studies. Pain Physician 2015;18:29-36.

17. Heavner JE, Racz GB, Jenigiri B, Lehman T, Day MR. Sharp versus blunt needle: a comparative study of penetration of internal structures and bleeding in dogs. Pain Pract 2003;3:226-31.

18. Akins EW, Hawkins IF Jr, Mladinich C, Tupler R, Siragusa RJ, Pry R. The blunt needle: a new percutaneous access device. AJR Am J Roentgenol 1989;152:181-2.

19. Quintero N, Laffont I, Bouhmidi L, Rech C, Schneider AE, Gavardin T, et al. Transforaminal epidural steroid injection and paraplegia: case report and bibliographic review. Ann Readapt Med Phys 2006;49:242-7.

20. Özcan U, Şahin Ş, Gurbet A, Türker G, Özgür M, Çelebi S. Comparison of blunt and sharp needles for transforaminal epidural steroid injections. Agri 2012;24:85-9.

21. Shin J, Kim YC, Lee SC, Kim JH. A comparison of Quincke and Whitacre needles with respect to risk of intravascular uptake in S1 transforaminal epidural steroid injections: a randomized trial of 1376 cases. Anesth Analg 2013;117:1241-7.

22. Hong J, Jung S, Chang H. Whitacre needle reduces the incidence of intravascular uptake in lumbar transforaminal epidural steroid injections. Pain Physician 2015;18:325-31.

23. Smuck M, Paulus S, Patel A, Demirjian R, Ith MA, Kennedy DJ. Differential rates of inadvertent intravascular injection during lumbar transforaminal epidural injections using blunttip, pencil-point, and catheter-extension needles. Pain Med 2015;16:2084-9.

24. Nagpal AS, Chang-Chien GC, Benfield JA, Candido KD, Rana MV, Eckmann M. Digital subtraction angiography use during epidural steroid injections does not reliably distinguish artery from vein. Pain Physician 2016;19:255-66.

25. Hong JH, Huh B, Shin HH. Comparison between digital subtraction angiography and real-time fluoroscopy to detect intravascular injection during lumbar transforaminal epidural injections. Reg Anesth Pain Med 2014;39:329-32. 